\title{
COMMENTARY
}

\section{Fact vs Fallacy: The Anti-Vaccine Discussion Reloaded}

\author{
Lucas B. Stolle (D) - Rohit Nalamasu • Joseph V. Pergolizzi Jr. • \\ Giustino Varrassi (D) · Peter Magnusson · JoAnn LeQuang • \\ Frank Breve · The NEMA Research Group
}

Received: August 13, 2020 / Accepted: September 9, 2020 / Published online: September 23, 2020

(C) The Author(s) 2020

\section{ABSTRACT}

In the light of the COVID-19 pandemic, antivaccine sentiments have been on the rise, with a recent seminal study on the development of anti-vaccine views in social media even making its way into Nature Communications. Yet, with the current scientific consensus being in

Digital Features To view digital features for this article go to https://doi.org/10.6084/m9.figshare.12927965.

L. B. Stolle $(\bowtie) \cdot$ J. V. Pergolizzi Jr. · J. LeQuang ·

F. Breve

NEMA Research, Inc., Naples, FL, USA

e-mail: lucas.stolle@some.ox.ac.uk

R. Nalamasu

Department of Physical Medicine and

Rehabilitation, University of Nebraska Medical

Center, Omaha, NE, USA

G. Varrassi

Paolo Procacci Foundation, Rome, Italy

P. Magnusson

Centre for Research and Development, Uppsala

University, Region Gävleborg, Gävle, Sweden

P. Magnusson

Department of Medicine, Cardiology Research Unit, Karolinska Institute, Stockholm, Sweden

F. Breve

Department of Pharmacy Practice, Temple

University School of Pharmacy, Philadelphia, PA, USA overwhelming agreement over the safety and efficacy of vaccines, many scientists lose their grasp on the fears, concerns, and arguments that the opposition may hold. This paper discusses and evaluates vaccine-hesitant individuals on a socioeconomic, historical, and philosophical landscape. It also provides an analysis of common argumentative patterns and the psychological impact that these arguments may have on undecided individuals. The discussion also explores why anti-vaccine sentiments are on the rise, and how members of the scientific and medical community require a more structured approach to communicating key arguments. This is particularly important if vaccination rates and herd immunity are to be sustained. No longer is it sufficient to win arguments based on a factual and scientific basis, but rather scientists and medical practitioners have to focus on conveying confidence and reassurance on both an informative and emotional level to those with doubts and fears.

Keywords: Anti-vaccination movement; COVID19 vaccine; Immunization; Infectious disease; Mass vaccination; Vaccine; Vaccine hesitancy; Vaccination refusal 


\section{Key Summary Points}

The pro- vs anti-vaccine discussion has gained traction in the light of the recent COVID-19 pandemic.

In our discussion we review the antivaccine movement in its current state, including associated demographics, argumentative patterns, and putative developments.

We provide a discussion on the appeal of anti-vaccine sentiments from a psychological, historical, and philosophical point of view.

For physicians and healthcare practitioners we provide valuable approaches to engaging in confrontations with vaccine-hesitant individuals.

\section{DIGITAL FEATURES}

This article is published with digital features to facilitate understanding of the article. To view digital features for this article go to https://doi. org/10.6084/m9.figshare.12927965.

\section{INTRODUCTION}

While many believe that the anti-vaccine (antivax) movement is novel and a direct result of the increase of immunizations available, antivaccination sentiments are not a novel concept, and have actually been present as long as modern-day vaccines themselves $[1,2]$. Vaccination entered modern medical practice in the 1800s with the smallpox vaccine developed by Edward Jenner. Early vaccinations were rudimentary, as in the case of smallpox, where lymph was harvested from cowpox blisters of infected animals and injected subcutaneously into a healthy patient. As a result of the perception of danger as well as the use of animal products, the vaccine met objections from the clergy and those with a general distrust of medicine [2]. As mandatory vaccination of infants became codified into law with the Vaccination Act of 1853 and later 1867, some citizens showed resistance to what was perceived as interference into the medical decisions parents could make for children [3]. Other concerns included doubts of the safety and sanitation of early vaccination methods.

Vaccine hesitancy is not just an American phenomenon, however. Higher GDP countries have been shown to have the lowest confidence in vaccines, and countries like China (9\%), Austria (8\%), and Japan (7\%) have reported a significant proportion of unvaccinated population. Despite gaining significant traction [4], anti-vaccine sentiments are still in the minority, with estimates suggesting that about $80 \%$ of the world population agree that vaccines are safe [5]. Often, anti-vaccine viewpoints and arguments are more compelling [6] and almost as widespread in social media $[4,7,8]$, relative to their pro-vaccine counterparts, which could be contributing to the movement's rapid growth [9].

The current scientific consensus is in overwhelming support of the use of vaccines in children and that their benefits greatly outweigh their risks [10]. This is not to say that vaccines do not carry any risks. Adverse incidents with vaccines have occurred and raised serious concerns that brought about change. For example, the "Cutter incident" described by the Centers for Disease Control and Prevention (CDC) involved batches of the polio vaccine administered in 1955 that contained the live polio virus and resulted in many cases of paralysis $[2,11]$. History reports many vaccine safety issues, including the simian virus 40 in the inactivated polio vaccine and oral polio vaccine (1955-1963), Guillain-Barré syndrome in the swine flu vaccine (1976), intussusception and the RotaShield vaccine (1998-1999), glass contamination in the HPV vaccine (2013), and others [11].

Recent global struggles with COVID-19 infection have once again highlighted the importance of this topic. With the Moderna mRNA-1273 vaccine completing a phase 1 clinical trial with beneficial results in all 45 
patients [12] and currently starting a phase 3 trial [12], anti-vaccination sentiments and conspiracy theories have once again made their way into the spotlight.

\section{HISTORY OF THE MODERN ANTI- VAX MOVEMENT}

Safety concerns about vaccines have been around as long as there have been vaccines and many of these issues were valid and have led to corrective actions. But there are also currently still largely unfounded assertions from all over the world $[13,14]$ that vaccines are associated with autism spectrum disorder or neurological disorders and that vaccines contain harmful ingredients, such as thiomersal [15]. In the developed world, there is a profound sense of medical mistrust, along with concerns that repeated immunization protocols may be excessive, redundant, or profit-making enterprises rather than preventive medicine. Indeed, in the USA, vaccination development and production is transitioning more and more to the private from the public sector [16].

The anti-vaccination proponents, often called anti-vaxxers, are often caricatured in the media as uneducated, unsophisticated laypeople from lower socioeconomic strata [17], but demographic data find that those with vaccinehesitant thoughts and behaviors are more likely to be educated, older mothers with higher socioeconomic status [2]. The anti-vaccination proponents are a heterogeneous group; some want to avoid all vaccination while others want to avoid certain specific vaccinations or reduce or delay the overall number of vaccinations [2]. Benin and colleagues helped to define the subgroups of parents with respect to their ideological position on vaccination: accepters, vaccine-hesitant parents, late-vaccinators, and rejectors. Between the two extremes of accepters-who do not question vaccinations-and rejectorswho reject all vaccinations without discussion-fall those who have serious concerns about vaccinations which may cause them to delay, postpone, or selectively vaccinate [18]. Benin's model may be a reductionist approach in that it does not offer a group for parents who question vaccination but then arrive at the decision to vaccinate based on their own research and conclusions that the benefits of vaccination outweigh the risks. Nevertheless, it demonstrates that some anti-vaxxers are following the paradigm of shared decision-making and weighing the risks and benefits, although their own distrust of modern medicine or other biases may come into play. Thus, the antivaccine community is to a large extent the product of educated individuals who have the capacity for critical thinking and a desire to discuss the subject with their physician. Yet, the general attitude of the scientific literature towards anti-vaxxers may appear hostile and encourage their censorship [2,19-21]. A possible explanation for the stark and sometimes politicizing polarities between these two groups may be related to the arguments advanced by anti-vaxxers.

\section{THE ROLE OF POST HOC ERGO PROPTER HOC}

Anti-vaccine arguments are often grounded in anecdotal evidence [6, 15], which runs the danger of leading to post hoc ergo propter hoc fallacies [22] (literally translated to "after this, therefore because of this"). False correlations can lead to arguments that can be propagated as fact through anti-vaccine blogs, social media pages [6], and even news outlets [22]. A common example of the post hoc fallacy in the antivaccine community is the controversial link between autism and the measles, mumps, and rubella (MMR) vaccine [23]. By convention, the first round of the MMR vaccine is administered at around 12 months of age, which coincides with the age at which behavioral symptoms of autism are first noted [24]. This timing has led some to draw a connection between vaccines and autism that has yet to be scientifically established. Similar associations between vaccines and allergies [25] as well as vaccines and asthma [26, 27] persist in the anti-vaccine community, although they lack credible scientific support. 


\section{THE ROLE OF BIAS}

There are several types of biases that can affect judgment. There are biases of omission (believing something despite a lack or paucity of evidence); confirmation bias (favoring arguments that support one's pre-established position); false or imagined correlations between events; the "availability heuristic" which gives undue weight to certain events at the expense of others; and cognitive dissonance, in which perceived "conflicting evidence" may lead one to resolve the internal conflict by selecting an incorrect but seemingly less dangerous solution. Parental vaccine hesitancy has been correlated with information avoidance and omission bias $[28,29]$. There is also a naturalist bias in the anti-vax community, which maintains that vaccines are manmade and therefore unnatural, so that they confer an immunity that is different and inferior to natural immunity [30]. This is not to say that naturally acquired immunity has no special value; it may confer protection against other diseases later in life, such as mumps clearance possibly reducing the risk of ovarian cancer [31]. This tendency to prefer natural immunity over vaccines, even in the absence of specific evidence, skews risk perception. Vaccines have been largely successful in eradicating many diseases, leaving parents and providers little direct experience with these illnesses [32]. This provides the lay public and the healthcare professionals with far more examples of putative vaccine side effects than the disease itself. Vaccine decisions can be affected by single case experiences or very small samples of alleged vaccination side effects [33].

\section{THE ROLE OF MISTRUST}

Mistrust of modern medicine, "big pharma," and government institutions is another source of vaccine hesitancy [34]. The ongoing COVID19 pandemic has itself generated conspiracy theories about the origin of the virus, the nature of the illness, and the objectives of a possible vaccine [34]. Conspiracy theories may be defined as the belief that multiple actors have forged a secret agreement to achieve a hidden and likely malevolent goal [35]. Among recent anti-vax conspiracy theories are beliefs that Bill Gates may be using the SARS-nCoV-2 virus vaccination to microchip the population or that the Gates Foundation is pursuing an agenda aimed at population control [34].

The mistrust of pharmaceutical companies is not entirely unfounded given the price-driving efforts of drug companies in the USA and recent findings about how oxycodone was marketed to vulnerable populations and led to the opioid crisis. The public's feeling of being at the mercy of larger well-funded organizational forces can create profound distrust, feed disillusionment, and fuel conspiracy theories. However, the antivax movement may have more to do with mistrust of institutions than specific conspiracy theories. For example, a lack of trust between a new mother and her child's pediatrician may play a major role in the decision-making process about vaccination [18]. Distrust appears to be stronger than trust, in that distrust in conventional medicine more strongly predicts vaccine hesitancy than trust in alternative or natural health remedies [36]. The level of information parents have about vaccines may play a large role in winning patient trust [37], and the availability of sound information, including patient-initiated research efforts, can help overcome doubts [38]. However, much of the available information is generated and supported by avid anti-vaxxers. This means that healthcare professionals must increase the quantity and quality of reliable, scientifically sound vaccine information online and accessible to patients. If this information was readily available, plentiful, and easily digestible to parents researching vaccinations, it would eliminate the need for parents to have to take "leaps of faith" with the acting pediatrician or other healthcare professionals [38].

\section{DENIALISM}

Denialism has been defined as rhetoric employed in order to give the appearance of legitimate debate where there is none, with the goal of rejecting an argument for which there is a consensus of expert opinion [39]. Pa Diethelm 
and Martin McKee assigned five qualities to denialism: conspiracy theories, "fake" experts, selective consideration (or exclusion) of evidence, impossible expectations of research, and misinterpretation of data and other logical fallacies [39]. Many vaccine-hesitant individuals can be placed somewhere on this spectrum. The exceptions are what one might call fair-minded skeptics, those individuals who out of a sense of responsibility for their child demand a full and complete presentation of data and objective professional expertise before reaching a decision.

The distinction between denialists and fairminded skeptics is important because these two groups process information differently, ask different sorts of questions, and make different types of arguments. A denialist, for example, may misinterpret data and study results but adamantly resist correction, while a fair-minded skeptic may demand the data but will discuss it openly and dispassionately. A fair-minded skeptic would revise his/her position on the basis of reliable information while a denialist would not.

\section{THE ROLE OF SOCIAL MEDIA}

While the role of confirmation bias in vaccine hesitancy and anti-vax sentiment remains disputed $[29,33]$, social media tends to artificially generate confirmation bias by its unilateral presentation of certain viewpoints [40]. Such bias may extend beyond user-generated social media to mainstream and other news media outlets [41]. Search engine algorithms and other editing algorithms tend to filter a user's internet experiences on the basis of prior searches and interactions. Thus, a person who has searched for anti-vax topics may in future searches get more or similar anti-vax information, creating a bubble of artificial confirmation bias that limits their exposure to alternative opinions [42].

A study of this sort of algorithm-driven social media ecology was offered by Johnson and colleagues about a burgeoning anti-vax tendency on Facebook [4]. According to their seminal study, anti-vaxxers are better positioned on Facebook to target clusters of vaccineneutral users and do so with greater efficiency than pro-vax groups [4]. On the basis of mathematical models of the spread of anti-vax information within the primary network patch, the authors have speculated that anti-vax viewpoints will predominate within the next 10 years [4]. This should not be surprising, as evidence suggests that anti-vax information is very attractive to vaccine-undecided individuals and these individuals can be swayed to make a lasting ideological change against vaccinations in 5 to $10 \mathrm{~min}$ [9]. Vaccine-skeptic individuals may lack the ability to critically assess online medical information. In a study of 34 vaccineundecided students presented with 40 websites containing varying degrees of factual medical information about vaccinations, 59\% of those in the study believed all of the websites contained accurate data when, in reality, only 18/40 were accurate [43]. These findings can be contextualized to more open social media platforms, such as Twitter, where it has been estimated that about half of all tweets about vaccines convey an anti-vax viewpoint [8]. A similar analysis on YouTube found that $32 \%$ of vaccine-related videos were anti-vax in orientation in 2007 [7]. In 2019, YouTube demonetized anti-vax channels by removing their advertising, although the content was allowed to remain [44].

Some social media platforms have made attempts to correct misinformation or limit its spread. Since 2019, Pinterest no longer allows searches on its site for "vaccines," but this drastic step initially disallowed all search results including those offering high-quality information [45]. More recently, a direct link has been established with health authorities, such as the World Health Organization and the Centers for Disease Control and Prevention, for vaccine-related topics [46]. These measures and other steps taken to circumvent anti-vax content may have a direct effect on the model proposed by Johnson et al. in reducing vaccination misinformation [4]. 


\section{DISCUSSION}

Scientists are committed to truth and object to false or misleading information, and this necessitates a willingness to hold open discussions with people who hold opposing points of view. There is no other way to work through information and reach valid conclusions based on facts. For that reason, scientists must encourage frank, open, and frequent discourse with the anti-vaxxers, but it is important for scientists to understand different anti-vax viewpoints for the most productive and mutually beneficial conversations. This is especially true for debates with healthcare professionals with anti-vax tendencies, as their arguments often stem from a scientific basis, as opposed to a anecdotal approach. Healthcare professionals are the interface in terms of sharing vaccination information with patients, but there are few studies about vaccine hesitancy in healthcare providers. Vaccine hesitancy among patients has been much more thoroughly investigated and thus any subsequent recommendations by the authors of this manuscript are primarily focused on engagements with the general antivaccine populous.

Denialists, a large subpopulation of the antivax movement, are typically motivated by greed, ideology, eccentricity, and idiosyncrasy [39]. There are certain ground rules of scientific debate: (a) the ability to critically evaluate one's own as well as the opposing viewpoints, (b) the ability to consider all of the evidence rather than just portions of it, and (c) the application of logic to making arguments. Without these ground rules in place, it is impossible to debate a topic rationally [39]. However, denialists do not employ this approach. Denialism, which may have its roots deep in human nature, has invaded our current culture to the extent that it is difficult to fight against. Instead, Diethelm and McKee suggest that pro-vaxxers change the subject from vaccines to the tactics that are being employed. If one could demonstrate that the anti-vax argument is unfair, biased, lopsided, or distorting, it might help reveal that the arguments are not valid. Furthermore, by discussing "how" the arguments are made rather than the arguments themselves, it may gradually sensitize denialists to the nature of scientific discussion. In other words, if denialists could be made to see, for example, that they are not considering all of the evidence but only a portion of it, it might help facilitate a more reasonable conversation. However, scientists have always had to deal with flawed, illogical, and prejudiced arguments; this is nothing new to science. One trend that must be resisted is a growing call for censorship or the silencing of anti-vax voices [2, 19]. Even established, highcaliber journals and well-published colleagues have advocated "shutting down" the anti-vax arguments [4]. This defeats the purpose of science, which should be to promote ideas and remove uncertainty in the minds of people rather than silencing them.

The other concern is how the pro-vax community can present information in a fair, balanced, but palatable way that opens the door for discussion with both neutral parties and skeptics. The distrust of many for the medical establishment has deep roots and is not entirely unfounded; scientists should not be quick to dismiss it. Creating narrative stories can be a helpful tactic, as much as scientists tend to prefer data and dry facts. The anti-vax narrative is replete with anecdotal evidence and storylines [47] which have the tendency to be misinterpreted and liberally extrapolated. The resulting narratives have broad appeal [14], as they are more tangible to the general populous [11]. Storytelling can be effective communication and there is no reason that it cannot be used by pro-vaxxers without sacrificing scientific soundness and accuracy. Vaccines protect us against a variety of terrible and even fatal diseases and there is no reason that storytelling cannot be used to make the pro-vax arguments more persuasive and more "human" than merely clinical trial data. The more liberal use of narrative stories to describe and defend the provax viewpoint should be encouraged.

Vaccine hesitancy is increasingly a global phenomenon. Virtually unrestricted travel enables vaccinated and unvaccinated individuals alike to get into contact with a myriad of diseases and the animals that spread them. This raises the question of the extent to which an 
individual's liberty outweighs the collective's drive to contain, eradicate, and protect themselves from diseases, whose spread can be quenched by inoculation. Unfortunately, there is no consensus regarding this predicament. Yet, answers to this ethical dilemma may well provide us with a new social standard reform that can have implications on the employment, education, and travel sectors; equally, it may impact the perception of vaccinated and unvaccinated individuals in the public eye. Thus, mandatory vaccines would eliminate one of the WHO's "ten threats to global health in 2019" [48] and a major free-riding externality as and in itself [49]. On the other hand, the infringement on personal liberty and bodily integrity that is associated with mandatory vaccinations, as well as the public scrutiny that may be associated with vaccination refusal may border the transgression of some constitutional and human rights.

\section{Limitations}

This commentary is an opinionated reflection on the current state of the anti-vaccine movement and the debate as a whole. This article is based on previously conducted studies and does not contain any studies with human participants or animals performed by any of the authors.

\section{CONCLUSION}

The anti-vaccination movement is actually a promising feature of human development, as it demonstrates a widespread tendency of individuals to think critically and independently. This is, however, not to say that the arguments are correct, only that they indicate a desire on the part of individuals to research and reach independent conclusions about important health-related topics. Johnson and colleagues [4] present an excellent argument and mathematical model showing how anti-vax ideas can spread widely on social media. In order to sustain global vaccination rates sufficient to maintain herd immunity, scientists may have to adapt their approaches to research, while public health officials adapt their approaches to raising awareness to address the anti-vax movement. Specifically, scientists must learn how to expose logical fallacies and other problems with denialists, and public health experts must elevate the use of storytelling over data presentation. It is fear, misinformation, and mistrust that are driving the anti-vax movement and scientists, the healthcare profession, and public health must provide open discourse and fair, balanced, accurate information that can assure patients rather than reinforce their despair.

\section{ACKNOWLEDGEMENTS}

Funding. No funding or sponsorship was received for this study or publication of this article.

Authorship. All named authors meet the International Committee of Medical Journal Editors (ICMJE) criteria for authorship for this article, take responsibility for the integrity of the work as a whole, and have given their approval for this version to be published.

Disclosure. Lucas Stolle, Rohit Nalamasu, Frank Breve, Peter Magnusson and JoAnn Le Quang have nothing to disclose. Giustino Varassi is a Section Editor for Advances in Therapy. Further, to complywith the International Committee of Medical Journal Editors (ICMJE) requirements, he discloses the following relationships: Consultant/Speaker and Researcher for Abbott, Dompé, Menarini, Molteni, Pfizer, Shionogi. Joseph V. Pergolizzi is on the Editorial Board of Advances in Therapy. To comply with the International Committee of Medical Journal Editors (ICMJE) requirements, he discloses the following relationships: Consultant/Speaker and Researcher for US World Meds, BDSI, Salix, Enalare, Scilex, Pfizer, Lilly, Teva, Regeneron, Redhill, Grunenthal, and Neumentum.

Compliance with Ethic Guidelines. This article is based on previously conducted studies and does not contain any studies with human 
participants or animals performed by any of the authors.

Open Access. This article is licensed under a Creative Commons Attribution-NonCommercial 4.0 International License, which permits any non-commercial use, sharing, adaptation, distribution and reproduction in any medium or format, as long as you give appropriate credit to the original author(s) and the source, provide a link to the Creative Commons licence, and indicate if changes were made. The images or other third party material in this article are included in the article's Creative Commons licence, unless indicated otherwise in a credit line to the material. If material is not included in the article's Creative Commons licence and your intended use is not permitted by statutory regulation or exceeds the permitted use, you will need to obtain permission directly from the copyright holder. To view a copy of this licence, visit http:// creativecommons.org/licenses/by-nc/4.0/.

\section{REFERENCES}

1. Greenberg SB. 'Bacilli and bullets': William Osler and the antivaccination movement. South Med J. 2000;93(8):763-7.

2. Hussain A, Ali S, Ahmed M, Hussain S. The antivaccination movement: a regression in modern medicine. Cureus. 2018;10(7):e2919.

3. Weber TP. Alfred Russel Wallace and the antivaccination movement in Victorian England. Emerg Infect Dis. 2010;16(4):664-8.

4. Johnson NF, Velásquez N, Restrepo NJ, et al. The online competition between pro- and anti-vaccination views. Nature. 2020;582:230-3.

5. Franklin H. How much does the world trust medical experts and vaccines? Wellcome. 2019. https:// wellcome.ac.uk/press-release/how-much-doesworld-trust-medical-experts-and-vaccines. Accessed Aug 2020.

6. Shelby A, Ernst K. Story and science. Hum Vaccines Immunotherap. 2013;9(8):1795-801.

7. Keelan J, Pavri-Garcia V, Tomlinson G, Wilson K. YouTube as a source of information on immunization: a content analysis. JAMA. 2007;298(21):2482-4.

8. Benecke O, DeYoung SE. Anti-vaccine decisionmaking and measles resurgence in the United States. Glob Pediatr Health. 2019;6: 2333794 X19862949.

9. Betsch C, Renkewitz F, Betsch T, Ulshöfer C. The influence of vaccine-critical websites on perceiving vaccination risks. J Health Psychol. 2010;15(3): 446-55.

10. van der Linden S. Why doctors should convey the medical consensus on vaccine safety. Evid Based Med. 2016;21(3):119.

11. Centers for Disease Control and Prevention. Historical vaccine safety concerns: Cutter incident 1955. . CDC. 2020. https://www.cdc.gov/ vaccinesafety/concerns/concerns-history.html. Accessed 28 May 2020.

12. Jackson LA, Anderson EJ, Rouphael NG, et al. An mRNA Vaccine against SARS-CoV-2-preliminary report. N Engl J Med. 2020. https://doi.org/10. 1056/NEJMoa2022483.

13. Lahariya C. A brief history of vaccines and vaccination in India. Indian J Med Res. 2014;139(4): 491-511.

14. World Health Organization. Immunization coverage. WHO. 2020. https://www.who.int/news-room/ fact-sheets/detail/immunization-coverage. Accessed 28 May 2020.

15. Kajetanowicz A, Kajetanowicz A. Why parents refuse immunization? Wiad Lek. 2016;69(3 Pt 1): 346-51.

16. Griesenauer RH, Kinch MS. An overview of FDAapproved vaccines and their innovators. Expert Rev Vaccines. 2017;16(12):1253-66.

17. Tsige B. Anti-vaccine movement: the epidemic of stupid. DiggitMagazine. 2019. https://www. diggitmagazine.com/column/anti-vaccinemovement-epidemic-stupid. Accessed 2020.

18. Benin AL, Wisler-Scher DJ, Colson E, Shapiro ED, Holmboe ES. Qualitative analysis of mothers' decision-making about vaccines for infants: the importance of trust. Pediatrics. 2006;117(5):1532.

19. Arede M, Bravo-Araya M, Bouchard É, et al. Combating vaccine hesitancy: teaching the next generation to navigate through the post truth era. Front Public Health. 2018;6:381.

20. Marti M, de Cola M, MacDonald NE, Dumolard L, Duclos P. Assessments of global drivers of vaccine 
hesitancy in 2014-looking beyond safety concerns. PLoS One. 2017;12(3):e0172310.

21. Salmon DA, Dudley MZ, Glanz JM, Omer SB. Vaccine hesitancy: causes, consequences, and a call to action. Vaccine. 2015;33(Suppl 4):D66-71.

22. Petousis-Harris HA, Goodyear-Smith FA, Kameshwar K, Turner N. Fact or fallacy? Immunisation arguments in the New Zealand print media. Aust N Z J Public Health. 2010;34(5):521-6.

23. Gerber JS, Offit PA. Vaccines and autism: a tale of shifting hypotheses. Clin Infect Dis. 2009;48(4): 456-61.

24. Johnson CP, Myers SM. Identification and evaluation of children with autism spectrum disorders. Pediatrics. 2007;120(5):1183-215.

25. Koppen S, de Groot R, Neijens HJ, Nagelkerke N, van Eden W, Rümke HC. No epidemiological evidence for infant vaccinations to cause allergic disease. Vaccine. 2004;22(25-26):3375-85.

26. Timmermann CA, Budtz-Jørgensen E, Jensen TK, et al. Association between perfluoroalkyl substance exposure and asthma and allergic disease in children as modified by MMR vaccination. J Immunotoxicol. 2017;14(1):39-49.

27. DeStefano F, Gu D, Kramarz P, et al. Childhood vaccinations and risk of asthma. Pediatr Infect Dis J. 2002;21(6):498-504.

28. Pomares TD, Buttenheim AM, Amin AB, et al. Association of cognitive biases with human papillomavirus vaccine hesitancy: a cross-sectional study. Hum Vaccines Immunotherap. 2020;16(5): 1018-23.

29. Jiménez ÁV, Mesoudi A, Tehrani JJ. No evidence that omission and confirmation biases affect the perception and recall of vaccine-related information. PLoS One. 2020;15(3):e0228898.

30. Price $S$. Talk to patients about: vaccine immunity vs natural immunity. Tex Med. 2020;116(3):47.

31. Cramer DW, Vitonis AF, Pinheiro SP, et al. Mumps and ovarian cancer: modern interpretation of an historic association. Cancer Causes Control. 2010;21(8):1193-201.

32. Schwartz JL. "Model" patients and the consequences of provider responses to vaccine hesitancy. Hum Vaccines Immunotherap. 2013;9(12):2663-5.

33. Haase N, Schmid P, Betsch C. Impact of disease risk on the narrative bias in vaccination risk perceptions. Psychol Health. 2020;35(3):346-65.
34. Jolley D, Douglas KM. The effects of anti-vaccine conspiracy theories on vaccination intentions. PLoS One. 2014;9(2):e89177.

35. van Prooijen J-W, Acker M. The influence of control on belief in conspiracy theories: conceptual and applied extensions. Appl Cogn Psychol. 2015;29(5): 753-61.

36. Hornsey MJ, Lobera J, Díaz-Catalán C. Vaccine hesitancy is strongly associated with distrust of conventional medicine, and only weakly associated with trust in alternative medicine. Soc Sci Med. 2020;255:113019.

37. Díaz Crescitelli ME, Ghirotto L, Sisson H, et al. A meta-synthesis study of the key elements involved in childhood vaccine hesitancy. Public Health. 2020;180:38-45.

38. Tafuri S, Gallone MS, Cappelli MG, Martinelli D, Prato R, Germinario C. Addressing the anti-vaccination movement and the role of HCWs. Vaccine. 2014;32(38):4860-5.

39. Diethelm P, McKee M. Denialism: what is it and how should scientists respond? Eur J Public Health. 2009;19(1):2-4.

40. Carrascosa JM, Cuevas R, Gonzalez R, Azcorra A, Garcia D. Quantifying the economic and cultural biases of social media through trending topics. PLoS One. 2015;10(7):e0134407.

41. Elejalde E, Ferres L, Herder E. On the nature of real and perceived bias in the mainstream media. PLoS One. 2018;13(3):e0193765.

42. Pariser E. Beware of online "filter bubbles". TED Talks. TED 2011 Web site. 2011. https://www.ted. com/talks/eli_pariser_beware_online_filter_ bubbles?language $=$ en. Accessed 2 Jun 2020.

43. Kortum P, Edwards C, Richards-Kortum R. The impact of inaccurate internet health information in a secondary school learning environment. J Med Internet Res. 2008;10(2):e17.

44. Shu C. YouTube demonetizes anti-vaccination videos. Tech Crunch. 2019. https://techcrunch. com/2019/02/22/youtube-demonetizes-antivaccination-videos/. Accessed 2 Jun 2020.

45. PBS News Hour. How social media is trying to contain misinformation over vaccines. PBS. 2019. https://www.pbs.org/newshour/health/how-socialmedia-is-trying-to-contain-the-spread-ofmisinformation-over-vaccines. Accessed 2 Jun 2020.

46. Ozoma I. Bringing authoritative vaccine results to Pinterest search. Pinterest. 2019. https://newsroom. 
pinterest.com/en/post/bringing-authoritativevaccine-results-to-pinterest-search. Accessed 2 Jun 2020.

47. Romijnders KAGJ, van Seventer SL, Scheltema M, van Osch L, de Vries H, Mollema L. A deliberate choice? Exploring factors related to informed decision-making about childhood vaccination among acceptors, refusers, and partial acceptors. Vaccine. 2019;37(37):5637-44.
48. World Health Organization. Ten threats to global health in 2019. WHO. 2019. https://www.who.int/ news-room/feature-stories/ten-threats-to-globalhealth-in-2019. Accessed 2020.

49. Ibuka Y, Li M, Vietri J, Chapman GB, Galvani AP. Free-riding behavior in vaccination decisions: an experimental study. PLoS ONE. 2014;9(1):e87164. 